\title{
Platinum Resistance
}

National Cancer Institute

\section{Source}

National Cancer Institute. Platinum Resistance. NCI Thesaurus. Code C147558.

A finding indicating that a cancer continues to grow despite the intervention with platinum therapy. 